\title{
The First Social Media Engagement Analysis of Health in Malaysia
}

\author{
Afiq Izzudin A. Rahim ${ }^{1}$, Mohd Ismail Ibrahim ${ }^{1}$, Faizul Nizam A. Salim ${ }^{2}$, Mohd \\ Ariff Ikram Ariffin ${ }^{3}$ \\ ${ }^{1}$ Department of Community Medicine, School of \\ Medical Science, Universiti Sains Malaysia, Kelantan, Malaysia; ${ }^{2}$ Director General's Office, Ministry \\ of Health, Putrajaya ${ }^{3}$ Corporate Communication Unit, Ministry of Health, Putrajaya, Malaysia
}

\begin{abstract}
Objectives: This study aimed to determine Facebook post characteristics and factors associated with good engagement rates among netizens on the Facebook (FB) page of Ministry of Health Malaysia.

Methods: 2123 FB posts were randomly selected in this cross-sectional study. The logistic regression model was applied to identify factors associated with good engagement rates.

Results: Majority of the FB post characteristics were organizational promotion content, photo type and posted between midnight and early morning. An FB post with a good engagement rate was significantly associated with a health education post (Adjusted Odd Ratio (AOR): 3.80, 95\% Confidence Interval CI: $3.02-4.78, \mathrm{p}<0.001$ ), a risk communication post (AOR: 1.77, 95\% CI: 1.39-2.26, p < 0.001), a post in the afternoon (AOR: 1.76, 95\% CI: 1.34-2.31, p < 0.001 ) or in the evening (AOR: $1.48,95 \% \mathrm{CI}: 1.20-1.82, \mathrm{p}<0.001$ ), and a video format (AOR: 3.74, 95\% CI: $1.44-9.71, \mathrm{p}=0.007)$.
\end{abstract}

Conclusion: We present the first comprehensive analysis of social media engagement and health communication analysis in Malaysia. The dynamic of health communication and rapid changes of health technology recently require health organizations to constantly upgrading their health promotion capacity and facilities. Therefore, they can effectively disseminate quality health information, achieving better audience engagement and subsequently improving health literacy among netizens on social media.

Keywords: social media, health information, health communication, health promotion. 